\title{
Die Kultur der Organisationsverbesserung in einer Abteilung für Innere Medizin
}

\author{
Die wichtige Innovation des Projekts «Domino» zur Optimierung der spitalinternen \\ Organisation war die direkte Beteiligung der Ärzteschaft an der Projektleitung. \\ Ausgehend vom Ist-Zustand wurden gemeinschaftlich Verbesserungspotentiale \\ gesucht und in Arbeitsgruppen die Wege zur Optimierung erarbeitet. Mit dem \\ Ergebnis sind alle mehr als zufrieden.
}

\author{
Olivier Giannini ${ }^{a}$, \\ Brenno Balestra ${ }^{b}$, \\ Graziano Selmonic, \\ Alberto Gandolfi ${ }^{d}$ \\ a Stellvertretender Chefarzt \\ Abteilung Innere Medizin \\ b Ärztlicher Leiter und \\ Chefarzt Abteilung Innere \\ Medizin \\ c Verwaltungsdirektor \\ «Ospedale Regionale di \\ Mendrisio Beata Vergine» \\ (OBV), Mendrisio \\ d Professor bei der Fachhoch- \\ schule «Scuola Universitaria \\ Professionale Svizzera \\ Italiana» (SUPSI) und Partner \\ von AFG Management \\ Consulting, Camorino, \\ Schweiz
}

\section{Die Herausforderung}

Das Regionalspital Mendrisio «Beata Vergine» (OBV) ist ein fachübergreifendes Spital, das mit den anderen Spitälern des Kantons Tessin im Netz des multilokalen Spitals «Ente Ospedaliero Cantonale» (EOC) vereint ist. Der OBV zählt etwa 400 Mitarbeiter. Die Abteilung für Innere Medizin verfügt über 64 Betten und ein Ärzteteam mit 21 Ärzten (11 Assistenzärzten) und 71 Krankenpflegern.

Wie in vielen anderen Schweizer Spitälern, hat sich auch im OBV das Problem der Überbelastung der Assistenzärzte ergeben. Nach dem Universitätsabschluss finden sich die Assistenzärztinnen und -ärzte in einem komplexen beruflichen Umfeld wieder, das sehr unterschiedliche Eigenschaften im Vergleich zur Vergangenheit aufweist. Bereits 2009 hatte die Spitalleitung die Fakultät Kommunikationswissenschaften der «Università della Svizzera Italiana» (USI) damit beauftragt, die Auswirkungen dieser Änderungen auf die Erwartungen der Senior-Ärzte und des Pflegepersonals an die Assistenzärzte sowie ihre Arbeitsqualität zu analysieren (Projekt VALMEDAS: Valorizzazione del lavoro dei medici assistenti: logiche d'azione e di giudizio a confronto all'OBV - Wertschätzung der Arbeit der Assistenzärzte: eine Gegenüberstellung der Handlungs- und Urteilsmethodik im OBV). Aus dieser Analyse haben sich erste Ideen und mögliche Verbesserungsmassnahmen ergeben, aber auch der Nachweis, dass die Berufs- und Ausbildungswelt, in der sich die Assistenzärzte bewegten, nicht zufriedenstellend war. Die Notwendigkeit, ein tiefgreifendes Umdenken zu starten und neue organisatorische Strategien und Verbesserungsmassnahmen auf mehreren Ebenen einzuleiten, hat sich deutlich gezeigt.

Dr. med. Olivier Giannini

Viceprimario Medicina Interna Ospedale Regionale della Beata Vergine CH-6850 Mendrisio Tel. 0918113227

Fax 0918113048

olivier.giannini[at]eoc.ch

\section{Die Ärzteschaft im Vordergrund}

Um diese Herausforderung zu bewältigen, hat sich die Leitung vom OBV für einen innovativen Ansatz entschlossen. Mit der Unterstützung eines externen Beraters wurde beschlossen, den methodischen

\section{Projet Domino: la culture de I'amé-}

\section{lioration de l'organisation dans}

\section{un service de médecine interne}

En 2010, un projet visant à améliorer et à optimiser l'organisation interne et les différents processus a été lancé à I'«Ospedale Regionale di Mendrisio Beata Vergine» (OBV). Ce projet, dont l'objectif principal était de décharger les médecins-assistants du service de médecine interne, a montré que les techniques de résolution de problèmes développées dans le milieu des affaires et appliquées à grande échelle peuvent également s'avérer efficaces dans le domaine hospitalier. Les mesures, définies par quatre groupes de travail interdisciplinaires, ont en effet permis des améliorations de taille. Trois mesures sont présentées ici: I'introduction d'une Memo Card visant à rationaliser les discussions de cas cliniques, l'élaboration d'un rapport de sortie clair et mieux structuré, et l'amélioration des processus de mise au courant et de formation des nouveaux médecins-assistants.

Un aspect essentiel et novateur a été la participation directe et enthousiaste des médecins aux questions touchant à la direction et à l'organisation. Ce facteur s'est montré décisif dans le succès de la nouvelle organisation et a permis de mener une discussion approfondie sur le leadership des médecins et la signification du dialogue interdisciplinaire au sein de l'hôpital.
Ansatz des Problem solving [1] und der ständigen Verbesserung [2] am Tätigkeitsbereich der Assistenzärzte anzuwenden. Es handelt sich um Techni- 


\section{Abbildung 1}

Das in unserem «Domino-Projekt» angewendete Problem-Solving-Verfahren. Mit «P» werden alle Plenarsitzungen angezeigt, in denen alle am Projekt beteiligten Mitarbeiter einen aktiven Beitrag zur Formulierung der Projekttätigkeiten leisten konnten

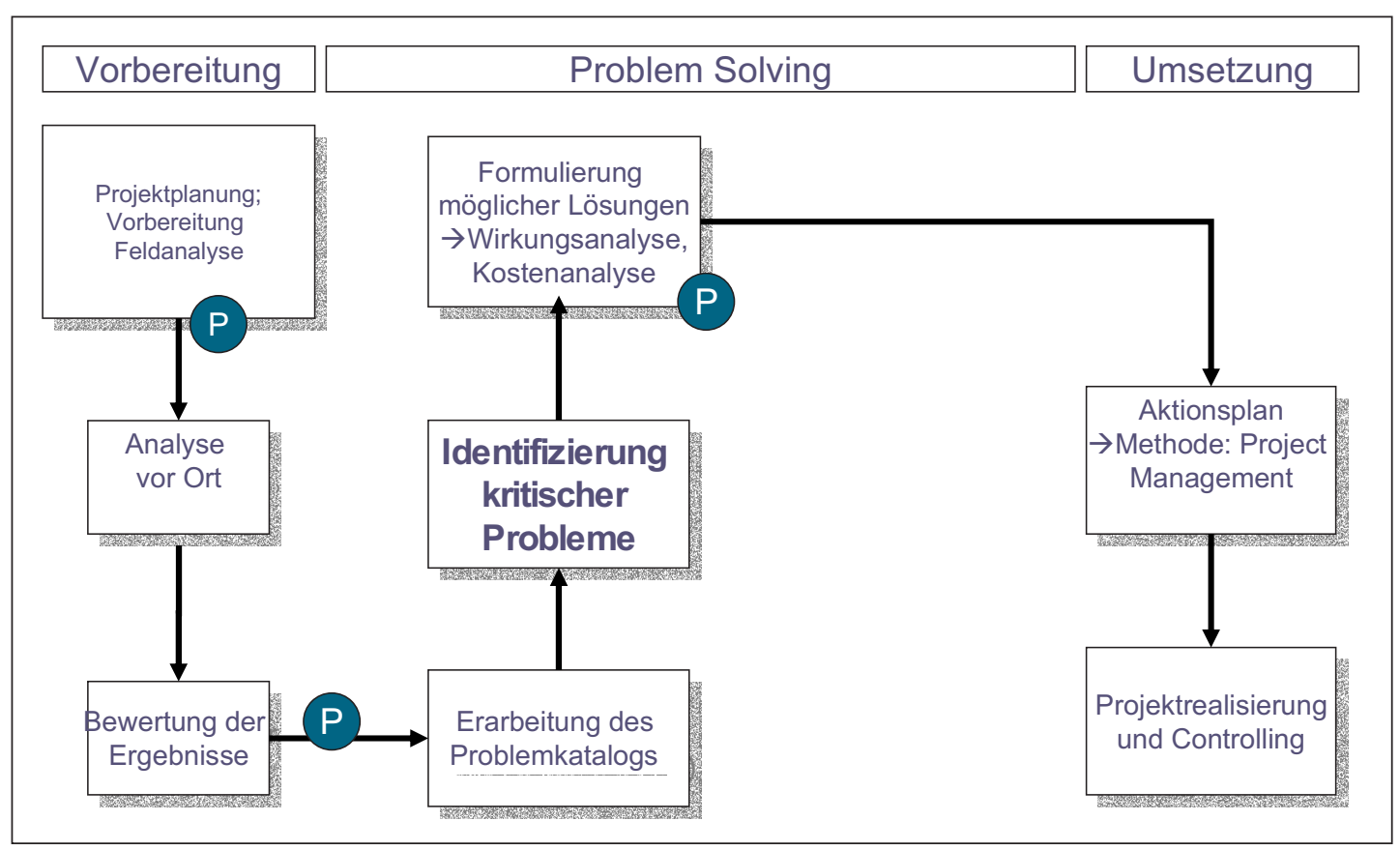

ken und Methoden, die seit mehreren Jahrzehnten in der Geschäftswelt angewendet werden, die seit kürzerem aber auch in zahlreichen Spitälern [3] vor allem im Bereich der Organisation, Verwaltung und Logistik umgesetzt werden. Der Einsatz auf dem Gebiet der effizienten Ärztearbeit ist aber noch selten [4]. Die Abbildung 1 zeigt den gewählten Projektweg und die Hauptaufgaben des Projekts. Das Projekt wurde «Domino» genannt, weil es als der erste Schritt eines umfassenderen Änderungsprozesses betrachtet wird, der später die anderen Abteilungen des Spitals und allgemein weitere organisatorische Prozesse des Spitals miteinschliessen sollte.

Das Ziel war nicht banal, da Ärztinnen und Ärzte zwar sehr offen für die technischen und relationalen Aspekte der medizinischen Tätigkeit sind, im Allgemeinen allerdings wesentlich weniger Interesse für die sogenannten Management-Aspekte ihrer Arbeit zeigen. Wenn wir aber die Welt der Ärzte aus einer neutralen Perspektive betrachten, wird uns bewusst, dass der Chefarzt ein echter «Leader» ist, der sein Team planen, leiten und motivieren muss. Die Teams können relativ gross sein und aus unterschiedlichen beruflichen Profilen bestehen, die in einem komplexen, äusserst dynamischen, unberechenbaren und fachübergreifenden Umfeld arbeiten. Das ist eben die Arbeit eines Managers. Die grosse Innovation des Projekts Domino war die direkte Beteiligung der Ärzteschaft an der Projektleitung. Die Projektleitung wurde vom stellvertretenden Chefarzt übernommen, der in enger Zusammenarbeit mit dem Chefarzt wirkte. Das ist wahrscheinlich der wichtigste Erfolgs- faktor für das gute Gelingen eines solchen Änderungsprojekts. Darüber hinaus wurde eine Vielzahl von Ärzten und Assistenten, Pflegekräften und Verwaltungspersonal an der Projektgruppe und an den verschiedenen Arbeitsgruppen beteiligt. Das Projektteam bildete den Kern und die «Lokomotive» der ganzen Initiative. Im Projektteam waren der Verwaltungsdirektor, der Chefarzt und der stellv. Chefarzt der Abteilung für Innere Medizin, die Verantwortlichen des Pflegepersonals, ein Vertreter des Controllings und der Personalabteilung des Spitals sowie ein Verantwortlicher der Revisionsstelle des EOC vertreten.

\section{Die Methode: organisatorisches Problem solving auch in der Medizin?}

Die Problem-Solving-Methode legt viel Wert auf die Analyse der Ausgangssituation: Erhebung von Daten und Indikatoren vor Ort, Wahrnehmungen der betroffenen Personen, Anzeichen von Problemen aller Art. Im OBV haben wir nach einer Vorbereitungsphase und nach der Bildung des Projektteams eine detaillierte Analyse der Arbeitszeiten der Assistenzärzte organisiert. Ein Expertenteam, das weitgehend aus Mitarbeitern des Controllings und der Revisionsstelle des EOC bestand, hat Minute für Minute die Arbeit der Assistenzärzte in fünfzehn unterschiedlichen Schichten verfolgt (Abteilung, Notaufnahme, Tag, Nacht und Wochenende). Obwohl die Datenmenge für eine wissenschaftliche Analyse nicht ausreichend war, konnte man feststellen, dass ein bedeutender Teil der effektiven Arbeitszeit für Neben- oder Verwaltungsauf- 


\section{Abbildung 2}

Beispiel für die Arbeitszeiterfassung der Assistenzärzte. Die Grafik zeigt die Arbeitszeiten während der Tagesschicht in der Abteilung (aggregierte Daten). Die Gewichtung der verwaltungstechnischen Aufgaben im Vergleich zum gesamten Arbeitsaufwand ist offensichtlich. Im Untersuchungszeitraum fand keine Aus- bzw. Fortbildung statt. Im Normalfall nimmt die Ausbildung 2 Stunden/Woche in Anspruch.

\begin{tabular}{|l|l|}
\hline Am Patientenbett \\
\\
\end{tabular}

gaben verwendet wurde. Während der Tagesschichten in der Abteilung wurde circa ein Drittel der Arbeitszeit für die Erstellung von Patientenunterlagen und Entlassungsbriefen aufgewendet (Abb. 2).

Bei der Erfassung der Arbeitszeiten haben die Beobachter auch eine Vielzahl von Anregungen für mögliche Verbesserungen gesammelt.

Parallel wurden weitere wichtige Daten erhoben. Das zentrale Sekretariat hat zwei Wochen lang das Erfassen und Versenden der Entlassungsbriefe untersucht und dabei festgestellt, dass die durchschnittliche Zeit zwischen der Entlassung eines Patienten
- Einführung der Assistenzärzte - Ausbildung und Arbeitsmethode

- Patientenbesuch - Schnittstelle mit Pflegepersonal - Schnittstelle mit Labor

- Organisation - Schnittstelle mit medizinischem Sekretariat - Verwaltung der Telefonanrufe

- Entlassungsbrief

Für die Auseinandersetzung mit diesen Themen wurden vier Arbeitsgruppen gebildet, die etwa zwei Monate lang intensiv daran gearbeitet haben. Im Rahmen einer abschliessenden Plenarsitzung haben alle Gruppen ihre Empfehlungen präsentiert. Diese wurden besprochen, kommentiert und von der Vollversammlung gebilligt [6]. Aufgrund dieser anregenden Diskussion hat das Projektteam einen Aktionsplan erarbeitet, in dem 36 Lösungsansätze, die jeweiligen Verantwortlichen und die Termine festgelegt wurden.

Ebenso wichtig war die darauffolgende Phase, nämlich das Controlling der Umsetzung. Viele Restrukturierungsprojekte scheitern gerade in der kritischen Phase der Umsetzung der Verbesserungsmassnahmen. Das Projektteam beschloss, sich einmal im Monat zu treffen, um die konsequente und systematische Umsetzung des Verbesserungsplans zu verfolgen und überprüfen. Das erste Treffen fand im September 2010 statt, und das Controlling wird heute noch fortgesetzt.

\section{Ergebnisse}

Wir möchten hier nur drei der zahlreichen Verbesserungsmassnahmen, die ab September 2010 in unserem Spital umgesetzt wurden, präsentieren.

\section{Die Vollversammlung hat dabei die Prioritäten gesetzt und die vorrangigen Punkte zusammengefasst.}

und dem Versenden des Entlassungsbriefes an den behandelnden Arzt ganze 27 Tage beträgt. Weitere relevante Abläufe (wie die Verarbeitung der Ergebnisse der Laboranalysen) wurden durch Process Management Tools untersucht.

Sämtliche Informationen haben zur Identifizierung des Verbesserungsbedarfs in den verschiedenen Bereichen beigetragen und die Suche nach möglichen Lösungen eingeleitet [5]. Alle Bemerkungen und Hinweise wurden vom Projektteam in 16 Kategorien aufgeteilt und im Rahmen einer Plenarsitzung mit allen betroffenen Mitarbeitenden besprochen. Die Vollversammlung hat dabei die Prioritäten gesetzt und die vorrangigen Punkte in vier Themenbereiche zusammengefasst:

\section{Memo Card}

Die Memo Card (Abb. 3) ist ein ideales Beispiel für eine Verbesserung, die scheinbar nur nebensächlich ist und praktisch zum Nulltarif umgesetzt werden kann, die sich aber entscheidend auf die ärztliche Kultur im Spital auswirkt. Es handelt sich um ein Blatt in einer Plastikhülle, das allen Ärzten und Assistenzärzten der Abteilung für Innere Medizin ausgeteilt wurde. Die Memo Card zeigt, welche Patientendaten bei der internen Berichterstattung präsentiert werden müssen. Es wurde nämlich festgestellt, dass vor allem die weniger erfahrenen Assistenzärzte dazu neigten, äusserst lange Präsentationen der Fälle zu machen und so einen wertvollen Zeitverlust für das gesamte Ärzteteam ver- 


\section{Abbildung 3}

Die Memo Card zeigt die wesentlichen Punkte, die den Kollegen für jeden klinischen Fall präsentiert werden müssen.

\begin{tabular}{|c|c|}
\hline \multicolumn{2}{|c|}{ Eingewiesen im Zimmer .... } \\
\hline Frau/Herr & (Vorname, Nachname) \\
\hline Alter & (Jahre) \\
\hline Für & (Grund der Einweisung) \\
\hline \multicolumn{2}{|c|}{ Nebendiagnosen } \\
\hline \multicolumn{2}{|c|}{ Unsere Diagnose oder Annahme } \\
\hline \multicolumn{2}{|c|}{ Therapeutische Massnahmen } \\
\hline Verlauf & \\
\hline
\end{tabular}

ursachten. Seit der Einführung der Memo Card weiss jeder Arzt genau, welche Aspekte wichtig sind, und die medizinischen Gespräche nehmen viel weniger Zeit in Anspruch. Die Zeit für die Präsentation eines Falles ist wesentlich kürzer geworden.

\section{Neuer Entlassungsbrief}

Der Entlassungsbrief ist ein äusserst wichtiges und heikles Dokument in der Kommunikation zwischen Spital und behandelndem Arzt bzw. anderen medizinischen Einrichtungen. Bei der Erfassung der Arbeitszeiten wurde festgestellt, dass die Assistenzärzte einen enormen Zeitaufwand für die Verfassung dieser Briefe benötigten. Die Arbeitsgruppe hat beschlossen, diese Situation in Angriff zu nehmen, und hat eine neue, kürzere und übersichtlichere Version des Briefes ausgearbeitet. Die neue Version wurde von den behandelnden Ärzten delnden Arzt gemessen. Dies bedeutet eine Reduktion um ca. 33\% im Vergleich zur Zeitmessung am Projektanfang.

Was den Zeitaufwand für die Verfassung des Briefes betrifft, stellten wir fest, dass die Verfassung eines kurzen und bündigen Briefes für viele Assistenzärzte eine beachtliche Aufgabe darstellt. Mit anderen Worten haben wir nachgewiesen, dass es viel leichter ist, alles zu schreiben als nur das Wesentliche. Die Einführung der neuen Version erfordert einen grösseren Aufwand bei der Kommunikation, Ausbildung und Begleitung der Assistenzärzte beim Erlernen der neuen Denkweise. Der neue Brief führte darüber hinaus zu einem unerwarteten Vorteil: Die Verrechnung der DRG-Pauschalen ist mit dieser neuen Version des Entlassungsbriefes wesentlich einfacher und schneller geworden.

\section{Einführung und Ausbildung der Assistenzärzte}

Die ersten Arbeitstage und Arbeitswochen sind eine schwierige Zeit für alle Assistenzärzte, vor allem wenn sie direkt nach dem Universitätsabschluss zu arbeiten beginnen. Eine Arbeitsgruppe hat die Abläufe und die Dokumentation der Einführungsphase untersucht und überarbeitet und eine vollständige Checkliste erstellt, mit der sämtliche in der Einführungsphase vorgesehenen Massnahmen systematisch überprüft werden können. Darüber hinaus wurden die Gestalt, die Rolle und die Verantwortlichkeiten des Tutors offiziell festgelegt. Es handelt sich dabei um einen SeniorArzt der Abteilung für Innere Medizin, der jeden Assistenzarzt, der die Tätigkeit im Spital aufnimmt, in Empfang nimmt, in die Arbeit einführt und begleitet. Der Tutor fördert, überprüft und zertifiziert die Umsetzung des Einführungsprogramms und der Ausbildungsziele der Assistenzärzte. Er unterstützt die Assistenzärzte und hilft ihnen bei der

\section{Für die Auseinandersetzung mit den wichtigsten Themen wurden vier Arbeitsgruppen gebildet, die zwei Monate lang intensiv gearbeitet haben.}

und den Kollegen der anderen EOC-Spitäler überprüft. Die Briefe sind jetzt kürzer und bündiger. Ausserdem wurden die Bedenken über die mangelnde Sicherheit des neuen Briefes ausgeräumt. In der Tat ergibt sich das Gegenteil: Wenn der Brief kurz gefasst ist und nur die wesentlichen Informationen enthält, ist die Wahrscheinlichkeit viel höher, dass er vom behandelnden Arzt vollständig gelesen wird.

Im März 2011 wurde eine erneute Zeiterfassung durchgeführt: Dabei wurde eine durchschnittliche Zeit von 18 Tagen zwischen der Entlassung des Patienten und dem Versenden des Briefes an den behan-
Ausarbeitung ihrer beruflichen Laufbahn, die im Jahresgespräch mit dem Chefarzt besprochen wird. Zwischen September 2010 und Juni 2011 profitierten 7 neue Assistenzärzte der Abteilung für Innere Medizin vom neuen Verfahren und von den neuen Einführungs- und Ausbildungsmassnahmen.

\section{Lessons learned: Was haben wir daraus gelernt?}

Wir betrachten das Projekt Domino als einen Erfolg. Im Mai 2011 konnte zum Beispiel festgestellt werden, dass die Assistenzärzte dank der Umsetzung der Verbesserungsmassnahmen am Morgen 
fast eine Stunde mehr Zeit für die Arbeit auf der Station und mit den Patienten zur Verfügung haben.

Allerdings ist wahrscheinlich der Weg, der zu diesen Verbesserungen und zur Stärkung der «ärztlichen Leadership» geführt hat, wichtiger als die erzielten Verbesserungen selbst. Im Projekt Domino haben die verschiedenen beruflichen Gruppen, die im Spital tätig sind, Seite an Seite an der Lösung konkreter Probleme gearbeitet. So sind sie sich bewusst geworden, dass Änderungen auch innerhalb einer eher «traditionellen» und wenig änderungsfreundlichen Struktur und Organisation möglich sind. Heikle und kom-

\section{Dank der Verbesserungsmassnahmen haben die Assistenzärzte morgens fast eine Stunde mehr Zeit für die Patienten und die Arbeit auf der Station.}

plexe Themen wie die Organisation der Arbeit in den Abteilungen am Vormittag wurden besprochen. Wie in einem Uhrwerk wirkte sich jede vorgeschlagene Änderung auf die Arbeit anderer aus, und daher war es sehr wichtig, einen offenen, direkten und konstruktiven Dialog zwischen allen Fachleuten (insbesondere zwischen der Ärzteschaft und dem Pflegepersonal) herzustellen, um gute Kompromisslösungen zu finden.

Mit dem Projekt konnten der interdisziplinäre Dialog und die fachübergreifende Zusammenarbeit im Spital und in der Abteilung für Innere Medizin gefördert und gestärkt werden - ein Erfolg, der sich positiv auf weitere Aspekte der Spitalarbeit auswirkt.
Gleichzeitig wurden sich alle bewusst, dass in einem Spital ein grosses «menschliches Vermögen» vorhanden ist und wie wichtig es ist, dieses enorme menschliche Potential mit seinen Ideen, Überlegungen, Bedenken und Vorschlägen zu nutzen, um die Qualität der Gesundheitsversorgung und das Leben der Menschen, die in einem Spital arbeiten, zu verbessern.

Ein relevanter Aspekt war die Überwindung des ursprünglichen Misstrauens der Ärzteschaft gegenüber Methoden und Ansätzen aus der «Managementpraxis». Diese haben sich aber als nützlich und angemessen erwiesen, um die alltäglichen Probleme der medizinischen Abteilungen in Angriff zu nehmen und zu lösen. Die Botschaft, die wir allen vermitteln wollten - und wir haben den Eindruck, dass diese Botschaft korrekt aufgenommen wurde - war, dass es möglich ist, einen Grossteil der Probleme, die täglich im Spital auftreten, mit einem strukturierten und professionellen Ansatz dank der Philosophie und den Mitteln der ständigen Verbesserung zu lösen.

\section{Literatur}

1 Alberti GE, Gandolfi A, Larghi G. La Pratica del Problem Solving. Milano: Franco Angeli; 2004.

2 Osterloh M, Frost J. Prozessmanagement als Kernkompetenz. Wiesbaden: Gabler; 1996.

3 Graban M. Lean Hospitals. New York: Productivity Press. Taylor \& Francis Group; 2009.

4 Pira A. Umfassendes Qualitätsmanagement im Spital. Zürich: VDF Hochschulverlag; 2000

5 Züst R. Einstieg ins Systems Engineering: Optimale, nachhaltige Lösungen entwickeln und umsetzen, Zürich: Orell Füssli; 2004.

6 Klebert K, Schrader E, Straub WG. ModerationsMethode. Hamburg: Windmühle Verlag; 2002. 